\title{
Erratum: Nucleon scattering on actinides using a dispersive optical model with extended couplings [Phys. Rev. C 94, 064605 (2016)]
}

\author{
E. Sh. Soukhovitskiĩ, R. Capote $\odot,{ }^{*}$ J. M. Quesada, S. Chiba, and D. S. Martyanov
}

(Received 2 December 2019; revised 7 August 2020; published 24 November 2020)

DOI: 10.1103/PhysRevC.102.059901

The dispersive optical model potential with the multiband coupling scheme and corresponding matrix elements derived in the original paper were implemented in a customized version of the FRESCO [1] code $^{1}$ to cross-check against the original calculations performed with the OPTMAN code [2,3]. It was found that optical model cross-section calculations presented in the paper were affected by coding errors in OPTMAN. The corrected version of the OPTMAN code calculates all cross sections in perfect agreement with FRESCO implementation.

The largest impact of the identified OPTMAN bugs on calculated neutron cross sections on even-even actinides is a 30-50\% overestimation of the coupling strength for the excitation of negative-parity bands (a smaller underestimation of the coupling strength of the "anomalous" $K=2$ nonaxial band was also found). Those coupling-strength differences lead to slightly larger inelastic cross-section values on corresponding coupled bands. Note that these changed cross sections still represent a small fraction of the total reaction cross section and do not affect noticeably the calculated total cross sections.

Due to OPTMAN coding errors, the optical model potential (OMP) parameters and parametrized coupling strengths had to be refitted and are tabulated in this Erratum. The following changes to the text are to be made.

(1) The nuclear shape used as a zero point for Taylor expansion of the optical potential is referred in the paper as "axially symmetric equilibrium shape." The word equilibrium should be dropped as the point of expansion of the optical potential does not necessarily correspond to the equilibrium nuclear shape; the Taylor expansion is performed around the axially symmetric rigid-rotor shape.

(2) Updated OMP parameters are slightly changed and are listed in Table I.

(3) These parameters have to be complemented by a corresponding coupling scheme (as published), and updated ground-state static deformations and coupling strength parameters (listed in Appendix A, Tables III-V, correspondingly.) to become a complete OMP parameter set for studied actinides.

The "effective deformations" in Tables IV and V correspond to those used in Eq. (B12) for even-even targets and Eq. (B16) for odd targets. Note that the code OPTMAN uses as input a different definition of "effective deformations" (labeled below with supraindex OPT), which are renormalized by a factor of $\beta_{20}$ compared to tabulated values and equations given in the paper, e.g., for $\left[\beta_{2}\right]_{\text {eff }}^{\mathrm{OPT}}$ we can write $\left[\beta_{2}\right]_{\mathrm{eff}}^{\mathrm{OPT}} \equiv \beta_{20}\left[\beta_{2}\right]_{\text {eff }}$ where the definition of $\left[\beta_{2}\right]_{\text {eff }}$ was given in the paper. Similar definitions apply to $\left[\gamma_{20}\right]_{\mathrm{eff}}^{\mathrm{OPT}},\left[\gamma_{22}\right]_{\mathrm{eff}}^{\mathrm{OPT}},\left[\beta_{30}\right]_{\mathrm{eff}}^{\mathrm{OPT}}$, and $\left[\beta_{32}\right]_{\mathrm{eff}}^{\mathrm{OPT}}$, respectively. In the original paper, we listed in tables the OPTMAN "effective deformations" (e.g., $\left[\beta_{2}\right]_{\mathrm{eff}}^{\mathrm{OPT}}$ ), whereas we prefer to list in the Erratum the true "effective deformations" $\left[\beta_{2}\right]_{\text {eff }}$. The different definition explains relatively large differences in value for effective deformations between the original paper and this Erratum.

(4) Optical model calculations with updated OMP parameters result in average resonance parameters shown in Table II; changes from published values are minor.

We would like to thank J. M. Franco-Patiño, A. M. Moro, and I. J. Thompson for their significant contribution to corrections discussed in this Erratum, and the work performed with the FRESCO code to verify the authors' calculations.

\section{APPENDIX: COUPLING SCHEME AND EFFECTIVE DEFORMATIONS FOR STUDIED ACTINIDES}

The updated tables in this Appendix correspond to those listed in Appendix $\mathrm{C}$ of the original paper.

\footnotetext{
*Corresponding author: r.capotenoy@iaea.org

${ }^{1}$ FRESCO implementation of the multiband coupling dispersive optical model potential will be published elsewhere.
} 
TABLE I. Dispersive coupled-channels optical model potential parameters for nucleon-induced reaction on actinides.

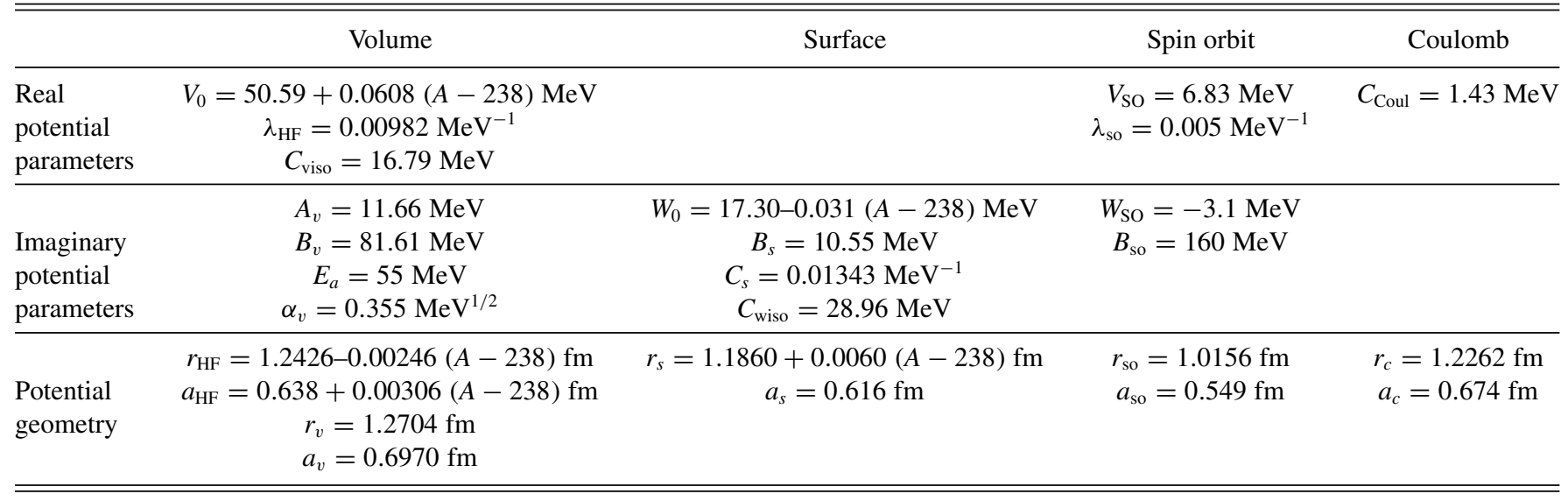

TABLE II. Calculations of average resonance parameters using derived OMP are listed. Strength functions are calculated at following incident neutron energies: ${ }^{232} \mathrm{Th}, 2 \mathrm{keV} ;{ }^{233} \mathrm{U}, 50 \mathrm{eV} ;{ }^{235} \mathrm{U}, 50 \mathrm{eV} ;{ }^{238} \mathrm{U}, 3 \mathrm{keV} ;{ }^{239} \mathrm{Pu}, 50 \mathrm{eV}$. Scattering radii are calculated at the incident neutron energy of $10 \mathrm{eV}$.

\begin{tabular}{lccrr}
\hline \hline Quantity & ${ }^{232} \mathrm{Th}$ & ${ }^{233} \mathrm{U}$ & ${ }^{235} \mathrm{U}$ & ${ }^{238} \mathrm{U}$ \\
\hline$S_{0},(\mathrm{eV})^{-1 / 2} \times 10^{-4}$ & 0.82 & 1.00 & 0.94 & 0.95 \\
$S_{1},(\mathrm{eV})^{-1 / 2} \times 10^{-4}$ & 1.84 & 1.59 & 1.57 & 1.67 \\
$R^{\prime}(\mathrm{fm})$ & 9.76 & 9.64 & 9.62 & 9.66 \\
\hline \hline
\end{tabular}

TABLE III. The ground-state (GS) deformation parameters of actinides allowing the best fit of experimental data using the updated OMP.

\begin{tabular}{lccr}
\hline \hline Target & $\beta_{2}$ & $\beta_{4}$ & $\beta_{6}$ \\
\hline${ }^{232} \mathrm{Th}$ & 0.201 & 0.074 & 0.00003 \\
${ }^{233} \mathrm{U}$ & 0.181 & 0.149 & -0.025 \\
${ }^{235} \mathrm{U}$ & 0.220 & 0.102 & -0.062 \\
${ }^{238} \mathrm{U}$ & 0.223 & 0.075 & -0.0062 \\
${ }^{239} \mathrm{Pu}$ & 0.226 & 0.092 & -0.005 \\
\hline \hline
\end{tabular}


TABLE IV. Interband effective coupling parameters and coupling scheme for $n / p+{ }^{238} \mathrm{U}$ (left side) and $n / p+{ }^{232} \mathrm{Th}$ (right side) reactions. The ground-state-band and the isobar-analog state (IAS)-band static deformations are the same as listed in Table III. Effective deformations $\left[\beta_{\lambda \mu}\right]_{\text {eff }}$ are defined after Eq. (B12).

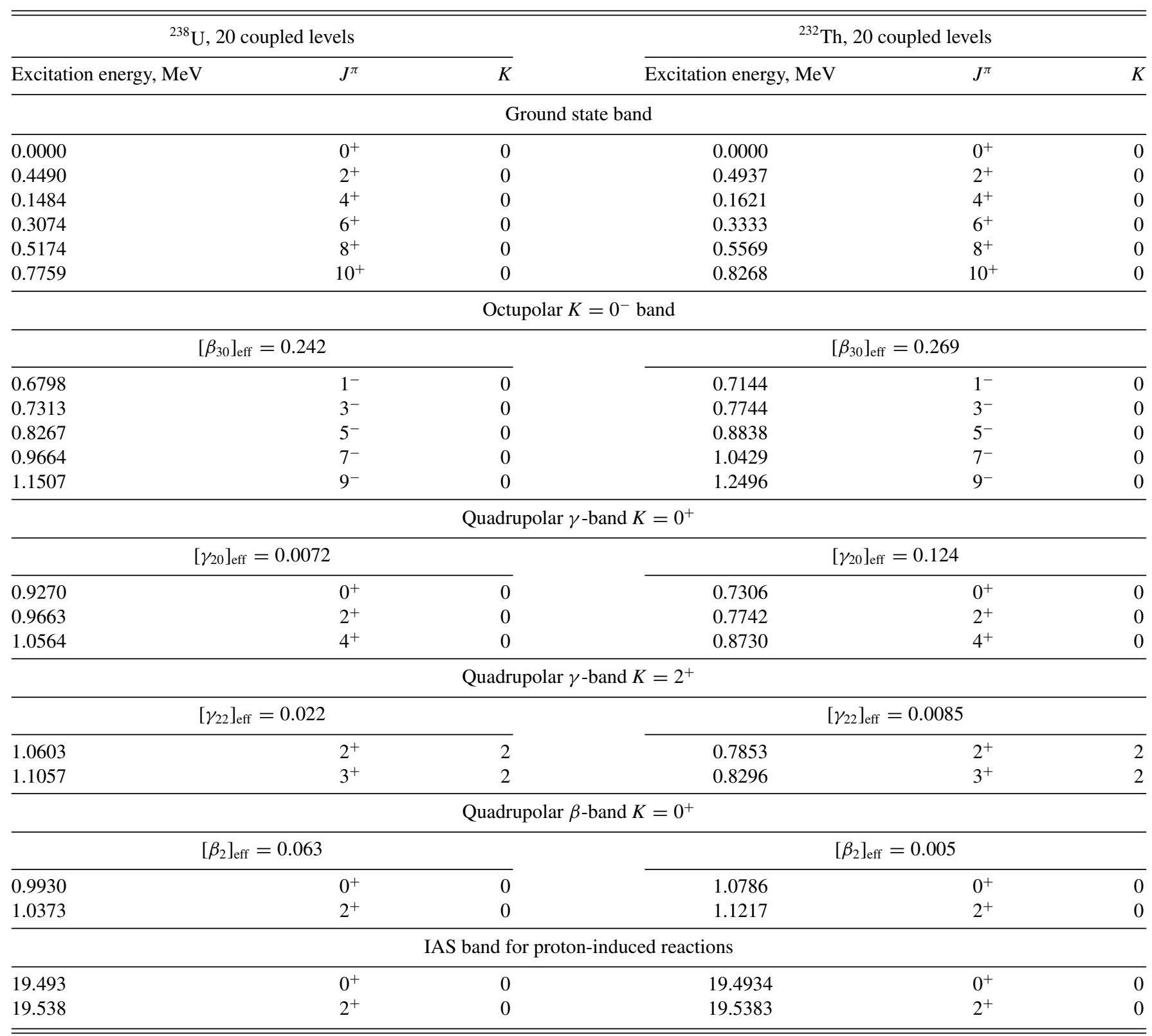


TABLE V. Interband effective coupling parameters and coupling scheme for $n / p+{ }^{239} \mathrm{Pu}$ (left side), $n / p+{ }^{235} \mathrm{U}$ (center), and $n / p+{ }^{233} \mathrm{U}$ (right side) reactions. The ground-state-band static deformations are the same as listed in Table III. Effective deformations $\left[\beta_{\lambda \mu}\right]_{\mathrm{eff}}$ are defined after Eq. (B16). Single-particle overlap factors $\alpha\left(v, v^{\prime}\right)$ are given by Eq. (B18).

\begin{tabular}{|c|c|c|c|c|c|c|c|c|}
\hline Excitation energy, $\mathrm{MeV}$ & $J^{\pi}$ & $K$ & Excitation energy, $\mathrm{MeV}$ & $J^{\pi}$ & $K$ & Excitation energy, $\mathrm{MeV}$ & $J^{\pi}$ & $K$ \\
\hline 0.00000 & $1 / 2^{+}$ & $1 / 2$ & 0.0000 & $7 / 2^{-}$ & $7 / 2$ & 0.00000 & $5 / 2^{+}$ & $5 / 2$ \\
\hline 0.00786 & $3 / 2^{+}$ & $1 / 2$ & 0.0462 & $9 / 2^{-}$ & $7 / 2$ & 0.04035 & $7 / 2^{+}$ & $5 / 2$ \\
\hline 0.16376 & $9 / 2^{+}$ & $1 / 2$ & 0.2491 & $15 / 2^{-}$ & $7 / 2$ & 0.22947 & $13 / 2^{+}$ & $5 / 2$ \\
\hline 0.19280 & $11 / 2^{+}$ & $1 / 2$ & 0.3385 & $17 / 2^{-}$ & $7 / 2$ & 0.31460 & $15 / 2^{+}$ & $5 / 2$ \\
\hline 0.31850 & $13 / 2^{+}$ & $1 / 2$ & 0.4394 & $19 / 2^{-}$ & $7 / 2$ & 0.41170 & $17 / 2^{+}$ & $5 / 2$ \\
\hline 0.35810 & $15 / 2^{+}$ & $1 / 2$ & 0.5512 & $21 / 2^{-}$ & $7 / 2$ & 0.51755 & $19 / 2^{+}$ & $5 / 2$ \\
\hline 0.51930 & $17 / 2^{+}$ & $1 / 2$ & & & & 0.63527 & $21 / 2^{+}$ & $5 / 2$ \\
\hline 0.49210 & $3 / 2^{-}$ & $1 / 2$ & 0.5106 & $9 / 2^{+}$ & $7 / 2$ & 0.32080 & $7 / 2^{-}$ & $5 / 2$ \\
\hline 0.50560 & $5 / 2^{-}$ & $1 / 2$ & 0.5788 & $11 / 2^{+}$ & $7 / 2$ & 0.35380 & $9 / 2^{-}$ & $5 / 2$ \\
\hline 0.55620 & $7 / 2^{-}$ & $1 / 2$ & 0.6902 & $13 / 2^{+}$ & $7 / 2$ & 0.39760 & $11 / 2^{-}$ & $5 / 2$ \\
\hline 0.58300 & $9 / 2^{-}$ & $1 / 2$ & & & & & & \\
\hline 0.66110 & $11 / 2^{-}$ & $1 / 2$ & & & & & & \\
\hline \multicolumn{9}{|c|}{ Quadrupolar $\gamma$ band } \\
\hline \multicolumn{3}{|c|}{$\alpha(5 / 2,1 / 2) \times\left[\gamma_{22}\right]_{\mathrm{eff}}=0.075$} & \multicolumn{3}{|c|}{$\alpha(3 / 2,7 / 2) \times\left[\gamma_{22}\right]_{\mathrm{eff}}=0.068$} & \multicolumn{3}{|c|}{$\alpha(1 / 2,5 / 2) \times\left[\gamma_{22}\right]_{\mathrm{eff}}=0.0061$} \\
\hline 0.28551 & $5 / 2^{+}$ & $5 / 2$ & 0.6378 & $3 / 2^{-}$ & $3 / 2$ & 0.39850 & $1 / 2^{+}$ & $1 / 2$ \\
\hline 0.33010 & $7 / 2^{+}$ & $5 / 2$ & 0.6645 & $5 / 2^{-}$ & $3 / 2$ & 0.41580 & $3 / 2^{+}$ & $1 / 2$ \\
\hline 0.38740 & $9 / 2^{+}$ & $5 / 2$ & 0.7012 & $7 / 2^{-}$ & $3 / 2$ & 0.45610 & $5 / 2^{+}$ & $1 / 2$ \\
\hline & & & 0.5332 & $9 / 2^{+}$ & $3 / 2$ & & & \\
\hline & & & 0.6082 & $11 / 2^{+}$ & $3 / 2$ & & & \\
\hline
\end{tabular}

[1] I. Thompson, FRESCO-Coupled Reaction Channels Calculations. Code and documentation available at www.fresco.org.uk

[2] E. S. Soukhovitskĩ̃, S. Chiba, O. Iwamoto, K. Shibata, T. Fukahori, and G. B. Morogovskiĩ, Programs OPTMAN and SHEMMAN Version 8, Technical Report No. JAERIData/Code 2005-002, 2005, available at https://doi.org/10.11484/ jaeri-data-code-2005-002.
[3] E. S. Soukhovitskiĩ, G. B. Morogovskiĩ, S. Chiba, O. Iwamoto, and T. Fukahori, Physics and Numerical Methods of OPTMAN: A Coupled-channels Method Based on Soft-rotator Model for a Description of Collective Nuclear Structure and Excitation, Technical Report No. JAERI-Data/Code 2004-002, 2004, available at https://doi.org/10.11484/jaeri-data-code-2004-002. 